\begin{tabular}{|c|c|}
\hline \multirow[t]{2}{*}{ 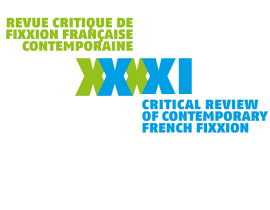 } & $\begin{array}{l}\text { Revue critique de fixxion française } \\
\text { contemporaine }\end{array}$ \\
\hline & $\begin{array}{l}21 \mid 2020 \\
\text { Fictions et pouvoirs }\end{array}$ \\
\hline
\end{tabular}

\title{
Samples pour un western spaghetti inspiré de nos vies
}

Fanny Taillandier

\section{(2) OpenEdition}

Journals

Édition électronique

URL : https://journals.openedition.org/fixxion/624

DOI : 10.4000/fixxion.624

ISSN : 2295-9106

Éditeur

Ghent University

Référence électronique

Fanny Taillandier, "Samples pour un western spaghetti inspiré de nos vies », Revue critique de fixxion française contemporaine [En ligne], 21 | 2020, mis en ligne le 15 décembre 2020, consulté le 17 février 2022. URL : http://journals.openedition.org/fixxion/624 ; DOI : https://doi.org/10.4000/fixxion.624

Ce document a été généré automatiquement le 17 février 2022.

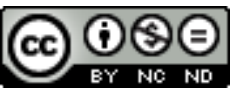

Les contenus de la Revue critique de fixxion française contemporaine sont mis à disposition selon les termes de la licence Creative Commons Attribution - Pas d'Utilisation Commerciale - Pas de Modification 4.0 International. 


\title{
Samples pour un western spaghetti inspiré de nos vies
}

\author{
Fanny Taillandier
}

"Tu vois, le monde se divise en deux catégories :
ceux qui ont un pistolet chargé et ceux qui
creusent. Toi, tu creuses."
Sergio Leone, Le bon, la brute et le truand, 1966

1 Je menais tranquillement ma Ford Fiesta vers ses $200000 \mathrm{~km}$ et la frontière, fermée, entre la France et l'Italie, quand l'autoradio me foudroya d'une évidence qui ne m'était jamais apparue: nous voudrions que notre vie se déroule sur une musique d'Ennio Morricone. L'examen attentif de nos désirs nous force à l'admettre. Nous aimerions, comme ça, que lorsque nous arrivons quelque part, le paysage se dévoile dans le fracas grandiose des cuivres. Qu'aux moments importants de nos vies, par exemple lors de rencontres déterminantes, un thème réapparaisse, à la fois discret et immédiatement reconnaissable, pourquoi pas joué par un harmonica ou alors par une de ces petites languettes qui font dong dong dong (des guimbardes peut-être). Une voix soliste pour nos amours, nos déchirures ; une envolée de cordes pour les trahisons, les haines, les vengeances et les victoires. Du moment que c'est signé Ennio Morricone.

2 Certes, nos vies ne ressemblent pas forcément beaucoup aux films qui ont rendu Morricone célèbre. Il est rare que nous jouions de la gâchette, le plus souvent faute de gâchette ; nul trésor ne nous attend quelque part dans l'Ouest, ou alors nous ne sommes pas au courant. Dans l'ensemble c'est plutôt des textos furax pour ce qui est des duels, et des crédits d'impôt sur l'isolation des vélux ou un CDI pas trop mal pour ce qui regarde la fortune. Mais malgré cela, nous ressentons exactement tout ce qu'expriment les instruments de l'orchestre, lorsque nous vivons. C'est pourquoi, aussi étrange que cela paraisse, nous aimerions bien que nos vies aient un titre qui commence par Il était une fois. Allons, disons-le, allons jusqu'au bout: nous aimerions bien être sur terre comme dans un film de Sergio Leone.

"Une voix me dit : Marche!"

Victor Hugo, Hernani, 1832 

croisant les doigts sur le volant de mon auto à l'approche de la douane, qui depuis quelques semaines faisait du zèle sur les cols des Alpes. Certes, dans le film, il y a beaucoup d'argent à la clé, largement de quoi remplacer la Fiesta par une élégante Lamborghini. Certes, Clint Eastwood est beaucoup plus classe que l'immense majorité des semblables qu'il nous est donné de côtoyer. Mais est-ce qu'on troquerait nos vélux et nos SMS contre ces vies de chiens? Honnêtement? Pas d'amour, des trahisons comme fonds de commerce, une hygiène déplorable. Tu t'apprêtes à manger la soupe en famille, un tueur déboule chez toi et t'abat pour trois-cents balles. Tu sauves la vie d'un type, il te laisse crever de soif dans le désert. Tu retrouves un ancien complice, il te passe à la question à te faire cracher tes dents. Tu n'as pas de maison, tu n'as pas de chemise de rechange, même à l'image on voit que tu sens. En plus, c'est la guerre de Sécession, et ça se met en travers de tous tes plans.

4 En effet, tous les retournements de situation dans l'intrigue - par ailleurs parfaitement hugolienne (c'est le célèbre tres para una, seulement appliqué à un sac d'or au lieu d'une femme) - sont liés à la guerre. Elle est présentée d'ailleurs comme une sanglante boucherie, et ce n'est plus le théâtre de Victor Hugo mais celui d'un Beckett historien : deux camps se battent pour un pont qu'il est interdit de détruire, alors qu'on peut traverser le fleuve avec l'eau à mi-poitrine; les morts s'amoncellent sans rime ni pardon; mortelle immobilité d'un casus belli inepte. Mais de cette rencontre improbable du romantisme avec l'absurde naît la magie du scénario de Sergio Leone : l'Histoire vient sauver le Bon d'une mort certaine par un boulet de canon perdu, au moment où le Truand allait le pendre ; faits prisonniers l'un et l'autre à cause d'uniformes pris comme déguisements, ils tombent sur la Brute, momentanément convertie en odieux gardechiourme, qui finalement les met sur le chemin de l'or. Et pour se sortir de la bataille du pont, quoi de plus malin que de le faire sauter, ce casus belli? "Alors, ces idiots iraient ailleurs pour faire la guerre".

5 Là où le western classique se proposait de moraliser le Far West, à grand renfort de sheriff et de veuves éplorées à sauver des Mexicains ou des Indiens, le western spaghetti rappelle que l'Etat n'a pas de morale. Il existe en revanche des individus qui font le choix du Good, d'autres celui du Bad, et l'immense horde des Ugly entre les deux, ces mélanges dégoûtants de blessures, de ridicule, de fourberie et de cruauté. Mais comment les juger? Tu es devenu prêtre, moi bandit, c'était les deux seules options que nous avions; et mon choix fut plus difficile que le tien, dit le Truand à son frère qui le rejette.

6 En s'emparant du western et en lui injectant un immoralisme à la fois tragique et comique, Sergio Leone fait autre chose que de très bons films. Il massacre de l'intérieur un genre narratif né pour chanter les louanges de la domination guerrière, et volontiers raciste, des Etats-Unis sur le territoire nord-américain. Il s'approprie une mythologie qui, pour être américaine, n'en est pas moins mondiale, celle de la conquête brutale présentée comme mission civilisatrice, et il la retourne, dévoilant la cruauté de son non-sens historique, où ne survit qu'une seule chose : la soif de vivre.

"Un bicot comme ça, ça nage pas! Haha, ça coule!"

Fonctionnaire de police en exercice à L'T̂le-SaintDenis (France), 26 avril 2020, après avoir repêché 
un homme qui s'était enfui en sautant dans la

Seine

7 Soit, mais tout cela ne nous éclaire pas beaucoup sur notre intime paradoxe, de désirer sourdement cette musique hyperbolique pour tout ce que nous vivons, pensais-je en tendant, soumise et apeurée, mon autorisation de passage aux flics des deux pays, puis en ouvrant le coffre de ma Fiesta les mains tremblantes, ce qui était déjà obéir à un abus de pouvoir. Pour l'héroïsme et l'intrépidité, je n'étais manifestement pas prête. Les cuivres de Morricone auraient certainement singé l'éclat de rire moqueur des mouettes - à moins qu'ils ne m'aient réconfortée d'une phrase explicitant la menace, parce que bon, si j'avais peur, j'avais sans doute des raisons pour.

Car peut-être ce désir dit-il ceci : que nous aussi avons l'impression que nos vies se font bouleverser par des guerres absurdes - on ne nous colle certes plus de baïonnettes dans les mains, du moins pour le moment; mais ce qui est certain, c'est que des genres de commandants d'armée ressemblant à des morts (comme le dit, dans le film, un tenancier d'hôtel voyant passer le général Lee) décrètent des choses qui viennent se mettre en travers de nos plans. Des frontières qu'on ferme du jour au lendemain, des statuts qu'on supprime, des droits qu'on escamote; tout un tas de changements de règles subits, et par nous subis. Nous avons des papiers, c'est bien. Nous avons la peau foncée, c'est moins bien. Nous n'avons pas d'autorisation, c'est vraiment pas terrible. Notre peau est sombre et nous souhaitons passer la frontière, c'est carrément la panade. Et notre soif de vivre passe son temps à chercher comment tourner ça à notre avantage, en tâchant de ne pas se retrouver au milieu d'une bataille quand on a besoin de passer le pont.

"Nous sommes en guerre", répétait d'ailleurs, dans les mêmes moments, un chef à l'apparence d'un mort. En premier examen, on était surtout enfermés chez nous. On ne risquait pas nos vies mais on s'ennuyait ferme; et en tournant sur les réseaux, en regardant nos stories, on tombait sur des vidéos, des témoignages qui montraient et racontaient qu'un homme plongeait dans un fleuve, déclenchant le rire et les insultes de soudards droits sortis du temps béni des colonies, qu'un jeune motard s'était fait arracher la jambe par la portière d'une voiture de flics, qu'une jeune fille se prenait un coup de Taser en pleine poitrine après avoir montré son attestation en rentrant des courses. Clairement, on restait chez soi.

"Il convient de rappeler que, face à des individus parfois délibérément récalcitrants au respect du confinement, la force a pu être légitimement employée pour maîtriser des personnes." Déclaration de la police nationale, Sud Ouest, 10 avril 2020

10 C'est drôlement bien dit. Ils parlent bien, les gens qui nous gardent, pensais-je en tâchant de remplir une nouvelle déclaration qui n'était pas dans ma langue maternelle, et dont mes années de latin classique (c'est-à-dire impérial, et il n'est pas anodin que les qualificatifs s'équivalent) ne m'aidaient guère à comprendre un traître mot.

11 "Il convient de rappeler", ça dit beaucoup, puisque nous en sommes à la grammaire. Une tournure impersonnelle qui annule la possibilité de toute alternative, puisque sans sujet, aucun autre sujet ne peut advenir. Et puis ce qu'il convient de faire, c'est de rappeler : l'histoire est déjà écrite, on était déjà censé la connaître, et si on a un doute c'est notre mémoire qui flanche. Nous, on avait pourtant nos stories qui partageaient à 
l'infini les insultes et coups reçus - la force légitimement employée - envers un livreur à mobylette, envers une ménagère, envers un employé de la logistique sortant travailler. C'était rageant.

12 C'est que les forces étatiques, quel que soit l'Etat, n'ont pas seulement le monopole de la violence légitime ; ils ont surtout pour eux la légitimité du récit, celle que donnent les armes. "Il convient de rappeler" de la langue administrative, celle qui selon Marshall McLuhan s'est développée en même temps que les routes de conquête de l'Empire romain, c'est le "il était une fois" d'une fiction qui ne s'embarrasse pas de s'avouer telle. Le moteur de l'auto toussotait dans les escarpements de Ligurie, du nom de ce peuple asservi par les Romains, et dont nous ne savons rien d'autre que ce qu'en disent les historiens latins, parce qu'ils n'utilisaient pas l'écrit - lesquels historiens les dépeignant d'ailleurs comme fourbes, voleurs, enfin tout ce que sont toujours les ennemis. "Il convient de rappeler" ouvre une fiction qui, par ses armes, par sa force, par son monopole, se rend classique - à enseigner en classe - et justifie a priori qu'on nous colle des baïonnettes dans les mains ou des coups de Taser dans le pif, selon les circonstances. Elle ne fait pas que cela, mais là n'est pas le propos: grâce à Sergio Leone, nous savons que l'histoire-qu'il-convient-de-rappeler, quoiqu'elle prétende, n'a pas de morale.

“Les vainqueurs l'écrivent, les vaincus racontent

l'histoire."

Booba, « 92i Veyron », Nero Nemesis, 2015 du dollar de Leone, du Professionnel de Verneuil, où Belmondo se faisait trahir par l'Etat français qu'il servait et se vengeait sur un dictateur allié de celui-ci. Le compositeur avait une formation classique, mais la popularité ne le dérangeait pas, et il injectait ses trouvailles de musique expérimentale dans les bandes originales qu'il composait. 
D'ailleurs, la musique populaire lui a rendu son affection : ses compositions ont été samplées dans plus de 400 morceaux de rap dans le monde.

"Dis, Tuco, tu aimes la musique? Ah oui, j'aime

Sergio Leone, Le Bon, la Brute et le Truand, 1966

16 Alors, quoi? Alors peut-être avons-nous de bonnes raisons de vouloir vivre comme dans Le bon, la brute et le truand : peut-être est-ce simplement parce que puisque nous ne sommes pas les vainqueurs (nous, à défaut de pistolet chargé, on est fatalement de ceux qui creusent) et puisque nous ne le serons jamais, nous avons du moins ce pouvoir de raconter à notre façon, de choisir nos héros, Good, Bad, Ugly, se jouant des turpitudes des empires soi-disant légitimes. Il y aura toujours, peut-être, des chefs à tête de mort téléguidant des Taser sur les frontières et sur les foules. Mais il y aura aussi, pour sûr, toujours des gens assoiffés de vivre, et d'autres pour raconter leur histoire avec orchestre symphonique parmi les gesticulations de ce-qu'il-convient-de-rappeler.

"Nouveau riche, ma Lamborghini a pris quelques dos-d'âne", chantait Booba dans le morceau précité, juste comme j'arrivais à Rome - et ma Fiesta aussi, qui faisait un nouveau bruit. Ce parrainage avec la Lambo dans la fiction, au fond, valait presque un trésor.

Je me souvins, en la garant dans cette ville de tant d'histoire, de trop d'histoire, de mon passage, juste avant la frontière, par cette vallée encaissée recelant des anciens lieux de culte ligures dont nous ne savons plus les légendes, et où des jeunes gens à la peau sombre bravent la police par les chemins de contrebande, passent en disant comme Ulysse que leur nom est Personne, pour se sortir de la panade historique et aller vers leur fortune. Puis j'allumai les stories, et je vis ce type anonyme escalader la façade haussmannienne qui fait face à la caserne de la garde, place de la République à Paris, à mains nues pour décrocher une insulte coloniale, sous les hourras de la foule multicolore. Nous avons nos personnages, nos héros, notre soif de vivre. Il ne nous reste plus qu'à sampler Morricone. 